\title{
Access to opportunities affects physics majors' interest and choice of methods specialization
}

\author{
Pedro Cardona, Dina Zohrabi Alaee, and Benjamin M Zwickl \\ Physics Department, Rochester Institute of Technology, 84 Lomb Memorial Dr, Rochester, NY, 14623-5604
}

\begin{abstract}
Physics is a degree that supports many career paths, and students often develop preferences for particular methods, such as theoretical, computational or experimental. However, it is not well understood how those preferences develop and affect students' later career decisions. We used Social Cognitive Career Theory (SCCT) as the basis for interpreting students' decision-making processes. SCCT provides a framework for connecting learning experiences, self-efficacy, and outcome expectations with students' interests, goals, and decisions. Semi-structured interviews with 8 physics students were conducted. This analysis focuses primarily on a single student to provide space to explore all three method specializations (theory, computation, and experiment) in more depth. We find that the availability of resources and learning opportunities had a significant impact on students' career choices. Theoretical and computational experiences were readily available through classwork, undergraduate research, and could be worked on at home and in peer study groups. Students lacked the ability to work on experimental physics outside of infrequent classroom opportunities and could not build peer networks that supported their experimental skill growth, which was linked to lower interest and self-efficacy in regards to experimental physics.
\end{abstract}




\section{INTRODUCTION}

A physics degree is a pathway to many different types of careers. Within physics, there are diverse specializations such as optics, astrophysics, particle physics, and biophysics, and within each of those subfields physicists often specialize in theoretical, computational, or experimental techniques. Although many students pursue graduate study, approximately $50 \%$ of physics majors seek employment after obtaining a Bachelor's degree, with the majority of those being computing and engineering related jobs in the private sector (i.e., not in academia, government, or other non-profit organizations) [1]. Within each of these different career paths, there are many decisions students must make.

The purpose of this paper is to use Social Cognitive Career Theory (SCCT) to examine the factors that contribute to the development of a physics student's specialization choices. Throughout this paper we use the term method specializations to refer to the three methods of physics that a student may choose to pursue (i.e., theoretical, computational, or experimental).

By performing interviews and analysis based in Social Cognitive Career Theory, we hope to gain a deeper understanding of how and why students gravitate towards particular specializations and career choices. This paper will concentrate on the decision of method specialization, though the data will show it is connected to other decisions a student may make, such as the particular subfield they choose to pursue. In the future, we hope to develop a physics-specific career decision-making survey that could be widely administered and focus on multiple decisions physics majors make in addition to method specialization.

\section{BACKGROUND}

Prior PER has used identity frameworks to study students' choice of physics as a major [3, 4]. However, we use Social Cognitive Career Theory to examine specific decisions that students make with the physics major experience. SCCT seeks to answer several questions about academic and career decisions, including how interests develop, how choices are made, and how success is achieved. It was developed in 1994 by Lent, Brown, and Hackett $[2,5]$ based on the social cognitive theory developed by Albert Bandura [6].

Social Cognitive Career Theory has been applied to understanding students' pursuit of STEM broadly [7, 8] and in specific STEM domains such as computer science $[9,10]$ and engineering $[11,12]$. For instance, Atadero et. al used SCCT to gauge if student interest in engineering was increased by introducing group design projects to a statistics course [12]. The study hoped to see if the introduction of group design projects made a significant impact on students' self-efficacy, outcome expectations, and goals. These three variables are the basic building blocks of SCCT, and they are linked together as shown in Fig. 1. Self-efficacy, outcome expecta- tions, and goals are the building blocks of SCCT because they are the parts of the SCCT map that change the most over time and between people. These constructs provide the most clear information for seeing how career paths develop.

Self-efficacy is a person's individual beliefs in their own abilities to perform a certain task or skill. It is not the same as a more broad confidence, as one may have stronger selfefficacy beliefs for a certain skill, such as math, but less for another skill, such as computing. In SCCT, it is assumed that people gravitate towards fields for which they have stronger feelings of self-efficacy.

Outcome expectations are beliefs about what a person expects will happen as a consequence of performing particular actions. SCCT assumes that people will engage in activities that they expect to be rewarding, whether that be socially, academically, or financially. For example, a physics major might attend research presentations because they expect to learn something about a field of research they are interested in pursuing. Even in the broader social cognitive theory, it is believed that engagement, effort, and ultimately success are at least in part determined by one's self-efficacy beliefs and outcome expectations.

In SCCT, goals are split into two distinct types, choice goals and performance goals [5]. Choice goals refers to an intent to take part in certain activities, while performance goals are an intent to achieve a certain level of success. Selfefficacy beliefs and outcome expectations both play an important role in setting goals. People are more likely to set goals that are in line with their self-efficacy beliefs, and that they expect to have a positive outcome. Harking back to the previous example, a physics student may make it a goal to attend a certain number of presentations related to their field of interest, or to reach out to the speaker to pursue a position as a research assistant.

Both self-efficacy and outcome expectations are informed by learning experiences. It is important to note that learning experiences come in many forms, not just classroom work. Learning experiences encompass all sorts of things, including classes, research experiences, hobbies, clubs, independent learning, or even vicarious experiences such as talking to a friend about a class.

\section{METHODOLOGY}

The interview protocol development and data analysis was significantly influenced by Social Cognitive Career Theory. The interview protocol was designed with distinct sections focusing individually on decisions regarding physics subfield, theoretical methods, computational methods, experimental methods, and grad school vs employment. Within each section, there were parallel sets of questions that covered the various constructs within social cognitive career theory (e.g., learning experiences or self-efficacy). Existing SCCT surveys and interview protocols provided some language to build upon $[9,13]$. 


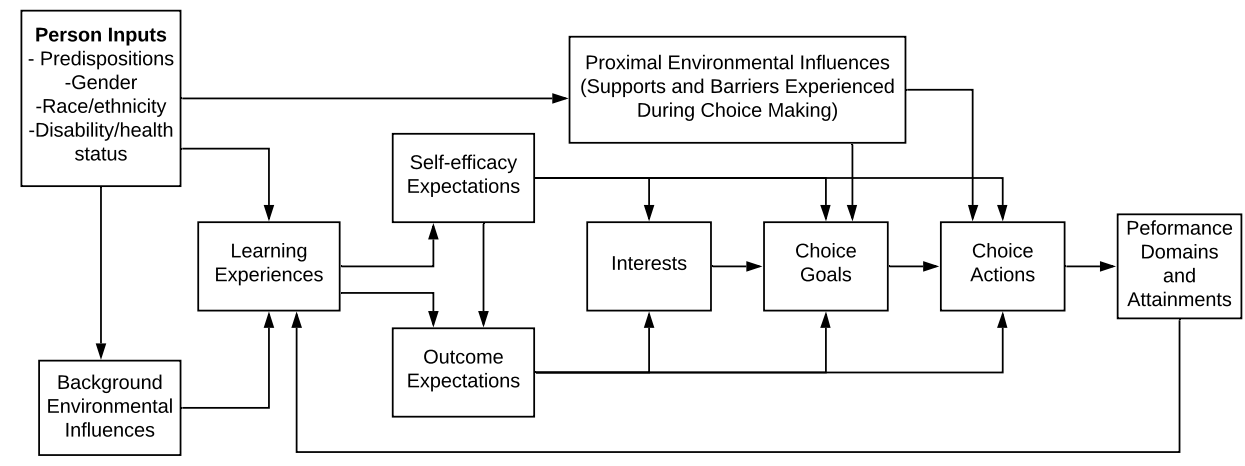

FIG. 1. Map of interrelated constructs within Social Cognitive Career Theory based on Lent, Brown, and Hackett (2002) [2]

An initial draft of the interview protocol was examined by both authors for alignment with SCCT constructs and the research question. Once the draft interview protocol was complete, two initial interviews were conducted to examine it for clarity of wording and to identify and improve places where it provided limited or irrelevant information. The structured nature of the interview helped the interviewer and participants settle into a rhythm of the questions. However, if the interviewer deemed that a particular question had been sufficiently addressed earlier in the interview it was skipped to improve the conversational flow.

Using the final version of the interview protocol, we conducted six additional interviews over Zoom. These interviews were conducted with students from two different universities. Each participant was a physics major in their 3rd or 4th year of college at the time of the interview. Participants were recruited through summer research programs and an online community of physics majors at one of the institutions. Six of the interviewees identified as male and two as female. Of the interviewees, four were Hispanic, one was African American, two were white, and one choose not to answer this question.

Each participant signed a consent form which explained the details of the project and asked for their permission to use their responses as part of the study. No participation incentives were provided. Most of the interviews ranged from 20 to 30 minutes, though two interviews lasted about an hour.

Initial transcripts were done using an automated transcription service integrated with Zoom, though researchers were required to make substantial corrections to the transcript before analysis. Qualitative data analysis of the transcripts was done in Dedoose [14]. Dedoose permitted collaborative coding of data when working remotely.

Each interview was initially coded into chunks based on the research question. Each chunk was then coded using a set of a priori codes for each SCCT construct shown in 1. A codebook was created to provide operational definitions of the SCCT constructs and to document the inclusion criteria and typical examples. For each instance where an a priori SCCT code was used, we also used Initial Coding to capture the essence of the excerpt [15]. Unlike the chunking and SCCT codes, the Initial Coding was mostly unique for each interviewee. A final round of analysis was used to organize the various SCCT and Initial Codes into a series of SCCT maps for each decision (e.g., theoretical physics), similar to Fig. 1 but with themes from the data filling in the specifics for each construct within the map.

For this work, we mainly focus on a student who we refer to as Paul. At the time of the interview, Paul was a Physics Major in his fourth year of college who identified as male and white. He gave his preferred subfield of physics as astrophysics and was intent on pursuing graduate studies. For the purpose of comparison, however, we also look at two other students, Mark and Felipe. Both were also fourth year Physics Majors. Mark identified as male and white, while Felipe identified as male and Hispanic.

\section{RESULTS}

The results are divided into three subsections based on Paul's responses to the three method specializations: theoretical, computational, and experimental. For each we describe how Paul's learning experiences and background have shaped his self-efficacy and outcome expectations, which further impact his interests, goals, and actions. These descriptions are based on SCCT maps created for each specialization. In Sec. IV C on experimental physics, we supplement Paul's views with those from Mark and Felipe.

\section{A. Theoretical}

When Paul was asked which of the three methods specializations he preferred, he answered "I would say theoretical." As the SCCT map in Fig. 1 suggests, Paul's interest in theory is linked to a moderate to high self-efficacy regarding theoretical work as well as positive outcome expectations. Paul's goals were influenced by an outcome expectation that very little astrophysics research was experimental. He explained that "it's a lot easier, a lot more applicable to use theoretical 
as opposed to experimental when you're talking about astrophysical ideas." He also believes in a general usefulness of theory. "If you have a theory and you can start from there, you could do what you need to do, whether you're making an experiment or writing code or even making more theories." When asked about his confidence in doing theoretical work, he answered that he was "pretty confident" but "not anticipating I'll do something incredibly, well, groundbreaking." This is a moderate level of self-efficacy; he feels confident, but not to the point of believing himself to be at the leading edge of the field.

Again, SCCT suggests those expectations and self-efficacy are affected by learning experiences. Paul says of his learning experiences, "the primary experience that I would have would be my own classwork but my Capstone [research experience] I'm working on definitely goes into the theoretical". From those experiences he gained confidence and came to the conclusion that theoretical work would be useful for his future career in astrophysics, as shown in the previous paragraph. These two things form the basis of his interest.

Going further in the SCCT map, after interest is choice goals. Paul states a goal of wanting to go to graduate school for astrophysics and "not end up with 40,000 dollars plus in debt." Again, we can see how the other parts of the SCCT map lead to this. To pay his student debt, he will need a job. And theoretical expertise is what he believes is most beneficial for a job in astrophysics, as well as for succeeding in graduate classes for astrophysics.

Finally, following along the map, we reach his choice actions, or initial steps taken to enact a career-decision. The student describes "looking at colleges, seeing what would be the best fit. And what would be the most advantageous down the line", and "having conversations with professors just helping me to get a good idea of what I should do."

\section{B. Computational}

Although Paul was most interested in theoretical physics, the interview protocol still included a series of questions about the other two method specializations, including computational physics. Paul acknowledged that while theory was his preference, he added afterwards "maybe a mix of computation. I'm definitely getting into computational a bit more." This is an interest which can be connected back to self-efficacy and outcome expectations.

For self-efficacy, when asked about his confidence in computational work, he said "when I came into college, not as confident, nowhere near as confident as I am now. I'm still definitely building confidence with it." He said his "coding experience before college was very, very low. Not nonexistent." This was followed by him saying, "Really only after, well, [a] computational physics [course] did I really get a taste and start using code consistently with different classes. I kind of expanded my knowledge base for code in conversation with my peers." This statement reveals how both for- mal coursework as well as informal interactions with peers have formed critical learning experiences. Elsewhere he also mentioned significant computational experience as part of his senior thesis project.

When asked about outcome expectations, the student answered that computational work is beneficial for his primary theoretical interest because "there's no clear cut 'you're only using of the three facets of physics" " and "at some point they all kind of blend together a bit."

Paul's choice goals for computational are the same as they are for theoretical because he sees the computational work as an essential complement to the theoretical work. Regarding specific choice actions, when given the chance to select a senior thesis project, he selected one with a combination of theory and computation.

In summary, Paul began with very little computational experience or knowledge. Through his learning experiences, he built up his self-efficacy in an area he thought would be useful to him in his pursuit of a career in astrophysics because of its close ties to theoretical work. Now, even though it is not his main pursuit or interest, he has developed more of an interest in it than when he started out.

\section{Experimental}

In contrast with theory and computation, which Paul has a strong interest in pursuing, he has no similar desire to pursue experimental work later after graduation. Again, SCCT provides an explanatory perspective. In terms of general interest, Paul mentions "It's not to say, I don't like experiments" but that theory is "a lot more applicable...as opposed to experimental when you're talking about astrophysical ideas." Paul has low outcome expectations regarding the usefulness of experimental skills and knowledge, which was evident with some humorous sarcasm to convey the difficulty in carrying out experiments in an astrophysics context: "Yeah, let me just make a black hole."

However, outcome expectations are not the full story. Selfefficacy was also lower as Paul acknowledges 'I'm not gonna say it's my best, but I feel confident enough that I would understand and be able to work with it." That moderate selfefficacy was built up was primarily through two junior level lab courses that cover a range of physics topics. "My main source of experience with experimental physics definitely coming from [two 3rd year lab courses]... and I think that's the same for a lot of us, besides [a senior research project]." Interestingly Paul does not mention introductory courses in mechanics and electricity and magnetism, which have a lab component integrated with the lecture nor a 2 nd year electronics course with two lectures and 1 lab each week.

Paul was not the only student with interest in experimental work. Two other students also expressed lukewarm or negative views. Felipe, a white Hispanic student entering his 4th year, described his interests as "Definitely theory. Theory and computational is alright, but like experimental, I don't want 
to be anywhere near an oscilloscope ever again in my life." After mentioning an interest in analyzing the data and comparing with theory, Felipe said " I don't know exactly what it is about doing the actual experiments. I was never particularly good at it and that was always like my weakest point, I think. Across all of my physics education has been actually performing the experiments. But, yeah I'm not sure exactly why. The concept of experimental stuff never really grabbed me like that." Felipe's low experimental self-efficacy goes beyond viewing it as weakness, but extends to a concern that he isn't sure what he could do to learn it better. Regarding outcome expectations, Felipe mentions a preference for theory because when reflecting on the larger research community he says, "I think that doing theoretical stuff is like really where we're learning new things." Although he acknowledges discovery happens using experiments, he believes it is more a part of theory.

Mark, who identified as a Latino or Hispanic male, also said his preference was "Anything except experimental." Mark specifically had a negative view about using experimental equipment. "I know a lot of people enjoy, like, doing the physics with their hands. I'm definitely not that guy. I'd rather just see it through equations." Mark expressed very positive views about theory and computation because "all my experience so far has been basically theoretical," which included a research experience with a theoretical professor. In terms of lower outcome expectations, Mark believed experimental work is burdened by slow feedback cycles compared to computation and theory where you can change parameters and see the response in a more rapid fashion. "In...experimental work you have basically one shot and then you have to restart experiments."

When considering Paul, Felipe, and Mark, all three students acknowledged less experience, lower self-efficacy, and lower outcome expectations for experiment relative to theory and computation.

\section{CONCLUSION}

The scope of this paper is limited, focusing on only three of eight three students interviewed, all of whom came from a single BS program. Ongoing work is aimed at including all eight students in the analysis, as well as expanding to include other physics undergraduate programs. Even within this limited scope, however, there is rich data to be analyzed.

Social Cognitive Career Theory applied to students' method specializations (i.e., theory, computation, experiment) has provided an insightful lens into physics majors' career decision making and was adaptable to these specific decisions and experiences faced by physics majors. The combination of self-efficacy and outcome expectations are readily identifiable, both in positive and negative characteristics influencing interest, actions, and decisions. When applying SCCT to students' narratives, it reveals a cyclical nature to the model. Actions taken by the student often become additional learning experiences, which may in turn further affect selfefficacy, outcome expectations, and therefore interest again. The path a student pursues because of their interests may lead them to an experience that opens them up to new interests.

Looking across the decisions related to experiment, theory, and computation, we see that the students analyzed in this paper all had the most learning experiences with theory and computation through classwork and research. These learning experiences left students with a positive impression about the flexibility of theoretical and computational knowledge and its relevance to scientific discovery in fields such as astrophysics. These substantial learning experiences supported a positive self-efficacy about future work in theory or computation.

Views on experiment were more problematic and mixed. Students noted fewer classroom experiences, and did not have research opportunities in experimental physics. Perhaps most concerning was Felipe's comment that experiment is a weakness, but he wasn't sure why or how to improve. Even if Felipe had a growth mindset, he lacked the resources, strategies, community, and role models to enact that growth. Experimental physics equipment, like Felipe's despised oscilloscope, is something that very few students have access to outside of their lab classroom. This means outside of a few hours per week in lab, they have very few low-stakes opportunities to practice, improve, and apply their experimental abilities. In contrast, theoretical learning experiences require only pencil and paper or whiteboards and markers. Similarly, computation has become widely accessible through open-source scientific computing tools (e.g., SciPy, R) and free cloud-based tools (e.g., Google Colaboratory). These tools permit students to engage in scientific computing at any time, in any class, or for self-study or a fun project.

This differential access to experimental opportunities suggests a greater need to support experimental opportunities outside of lab courses. Low-cost technologies such as Arduino microcontrollers or single board computer (e.g., Raspberry $\mathrm{Pi}$ ) open up a wide range of low cost experimental opportunities and other creative endeavors that could be accessible by students outside of the classroom. Similarly, electronics lab courses, which are common in physics departments, are amenable to outside of class exploration if courses adopt portable USB oscilloscope and waveform generators and lowcost electronics parts. Departmental makerspaces could be another strategy to support informal peer learning around experimental physics. Without such efforts, students who enter college without extra experience (e.g., robotics club or hobbies), may be at a significant disadvantage, which may lead to lower experimental self-efficacy throughout their undergraduate experience. Mark, who had a low view of experiment, acknowledged he was raised by a single mother and his childhood "was kind of poor. And so I just read a bunch...I would read books on like space science, stuff like that." The theoretical side of physics was most accessible during these early years and Mark still has a passion for those same things. More research and innovation needs to be done to ensure experimental physics is also accessible to all physics majors. 
[1] American Institute of Physics, Employment and Careers in Physics, Tech. Rep. (2020).

[2] R. Lent, S. Brown, and G. Hackett, in Career Choice and Development (4th Ed.) (Wiley, 2002) pp. 750-754.

[3] Z. Hazari, G. Sonnert, P. M. Sadler, and M.-C. Shanahan, Journal of Research in Science Teaching 47, 978 (2010), _eprint: https://onlinelibrary.wiley.com/doi/pdf/10.1002/tea.20363.

[4] R. M. Lock, Z. Hazari, and G. Potvin, Physical Review Physics Education Research 15, 020137 (2019), publisher: American Physical Society.

[5] R. W. Lent, S. D. Brown, and G. Hackett, Journal of Vocational Behavior 45, 79 (1994).

[6] A. Bandura, Social foundations of thought and action: A social cognitive theory, Social foundations of thought and action: A social cognitive theory (Prentice-Hall, Inc, Englewood Cliffs, NJ, US, 1986).

[7] H.-B. Sheu, R. W. Lent, M. J. Miller, L. T. Penn, M. E. Cusick, and N. N. Truong, Journal of Vocational Behavior 109, 118 (2018).

[8] W.-C. Mau, S.-J. Chen, and C.-C. Lin, Education Sciences 9,
151 (2019).

[9] R. W. Lent, A. M. Lopez, F. G. Lopez, and H.-B. Sheu, Journal of Vocational Behavior 73, 52 (2008).

[10] A. Alshahrani, I. Ross, and M. I. Wood, in Proceedings of the 2018 ACM Conference on International Computing Education Research, ICER '18 (Association for Computing Machinery, Espoo, Finland, 2018) pp. 205-214.

[11] R. W. Lent, H.-B. Sheu, D. Singley, J. A. Schmidt, L. C. Schmidt, and C. S. Gloster, Journal of Vocational Behavior 73, 328 (2008).

[12] R. A. Atadero, K. E. Rambo-Hernandez, and M. M. Balgopal, Journal of Engineering Education 104, 55 (2015).

[13] C. W. Bulgren, The Career Interests, Choices, And SelfEfficacy Of Male Elementary General Music Teachers, Ph.D. thesis, University of Michigan (2017).

[14] Dedoose version 8.3.47 (2020).

[15] J. Saldaña, in Coding Manual for Qualitative Researchers (SAGE Publications Ltd, 2013) 2nd ed. 